\title{
Identities and Relationships among Chinese Vegetable Brassicas as Determined by Random Amplified Polymorphic DNA Markers
}

\author{
Jianping Ren \\ Institute of Vegetables and Flowers, Chinese Academy of Agricultural Sciences, Beijing 100081 China \\ James R. McFerson \\ USDA-ARS Plant Genetic Resources Unit, Cornell University, Geneva, NY 14456-0462 \\ Rugang Li \\ Biotechnology Research Center, Chinese Academy of Agricultural Sciences, Beijing 100081 China \\ Stephen Kresovich \\ USDA-ARS Plant Genetic Resources Conservation Unit, University of Georgia, Griffin, GA 30223-1797
}

\author{
Warren F. Lamboy ${ }^{1}$ \\ Department of Horticultural Sciences and USDA-ARS Plant Genetic Resources Unit, Cornell University, \\ Geneva, NY 14456-0462
}

Additional index words. Brassica rapa, Brassica juncea, Chinese cabbage, pakchoi, genetic diversity, genetic resources collection

\begin{abstract}
Fifty-two germplasm accessions of Chinese vegetable brassicas were analyzed using 112 random amplified polymorphic DNA (RAPD) markers. The array of material examined spanned a wide range of morphological, geographic, and genetic diversity, and included 30 accessions of Brassica rapa L. (Chinese cabbage, pakchoi, turnip, and broccoletto), 18 accessions of $B$. juncea (L.) Czern. (leaf, stem, and root mustards), and four accessions of B. oleracea L. ssp. alboglabra (Chinese kale). The RAPD markers unambiguously identified all 52 accessions. Nei-Li similarities were computed and used in unweighed pair group method using arithmetic means (UPGMA) cluster analyses. Accessions and subspecies were clustered into groups corresponding to the three species, but some accessions of some subspecies were most closely related to accessions belonging to other subspecies. Values for Nei-Li similarities suggest that Chinese cabbage is more likely to have been produced by hybridization of turnip and pakchoi than as a selection from either turnip or pakchoi alone. RAPD markers are a fast, efficient method for diversity assessment in Chinese vegetable brassicas that complements techniques currently in use in genetic resources collections.
\end{abstract}

The crop brassicas comprise six economically important species with great genetic and morphological diversity. These plants yield edible roots, stems, leaves, buds, flowers, and seeds. In addition, some of the types are used as forage, sources of oil, or ornamental.

The origin, evolution, taxonomy, and genomic relationships of the crop Brassicas have been studied extensively. The elementary species are Brassica nigra Koch $(\mathrm{n}=8$, genome B), B. oleracea ( $n$ $=9$, genome $\mathrm{C})$ and B. rapa $(n=10$, genome $\mathrm{A})$, which are believed by some to be secondarily balanced polyploids (Prakash and Hinata, 1980). Brassica carinata A. Br. $(n=17$, genome BC), $B$. juncea $(n=18$, genome AB), and B. napus L. $(n=19$, genome AC) each originated by hybridization and polyploidization of two different elementary species (U, 1935).

Judging by the distribution of its wild forms, the genus Brassica probably originated in the Mediterranean-Middle Eastern area (Tsunoda, 1980). A secondary center of origin and differentiation

Received for publication 18 Oct. 1994. Accepted for publication 27 Dec. 1994. Mention of a proprietary product does not constitute an endorsement or recommendation for use by the U.S. Dept. of Agriculture. Chuck Simon, Michael Havey, and an anonymous reviewer offered valuable comments and criticism that considerably improved the manuscript. Anne Westman read the manuscript and provided many useful suggestions. This work was funded in part through a collaborative research project between China, USDA-OICD, and USDA-ARS, ARS Project 190821000-003-09T. The cost of publishing this paper was defrayed in part by the payment of page charges. Under postal regulations, this paper therefore must be hereby marked advertisement solely to indicate this fact.

'To whom reprint requests should be addressed. of the species B. rapa and B. juncea appears to be China (Nishi, 1980). Since their introduction into China thousands of years ago, these two species have been changed significantly in form, structure, and productivity by domestication. As a result of the allogamous breeding system in Brassica, there is a large amount of morphological variability in the many subspecies, botanical varieties, and cultivar groups of B. rapa and B. juncea (Lee, 1982; Li, 1981; Opena et al., 1988).

Brassica rapa ssp. pekinensis (Lour.) Olsson (Chinese cabbage) is the most popular and important vegetable crop in China. The leaves of the plant are usually eaten fresh, although sometimes they are preserved by fermentation. Although Chinese cabbage originated in China sometime before the 10th century (Li, 198 1), Brassica rapa ssp. rapifera (Metzg.) Sinsk. (turnip) in northern China and ssp. chinensis (L.) Makino (pakchoi) in southern China have been cultivated much longer, at least since the 5th century B.C. (Li, 1981). Li (1981) has suggested, therefore, that ssp. pekinensis originated in central China by hybridization between ssp. rapifera and ssp. chinensis, since the latter two subspecies were commonly grown together historically and had ample opportunity to interbreed. Alternatively, Ten ( 1980) proposed that ssp. pekinensis originated as a wild or semi-cultivated plant through differentiation of and selection from ssp. chinensis alone.

Like Brassica rapa, B. juncea (mustard) is a valuable vegetable crop in China, possessing a range of morphological variation in China that is even greater than that of Chinese cabbage (Lee, 1982). The stems, roots, and leaves of various cultivars of $B$. 


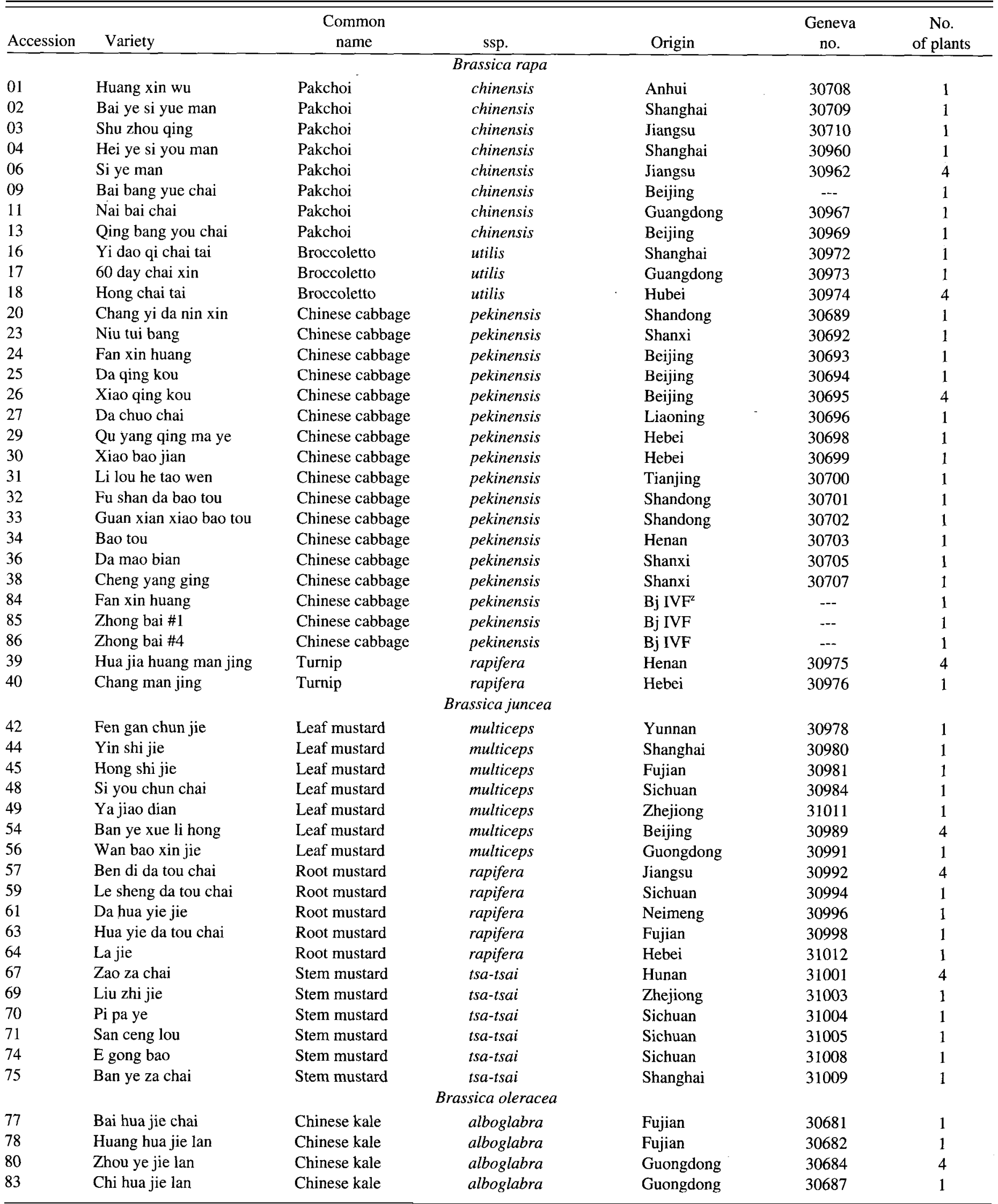

${ }^{\overline{ }}$ Beijing; Institute of Vegetable and Flowers of the CAAS. 
juncea are used as vegetables throughout the country. Unlike Chinese cabbage, mustard is used primarily in the fermented, preserved state and some is eaten fresh. The species is particularly important in the southern region (Nishi, 1980). For use as greens, it is second only to radish (Raphanus sativus L.) in Taiwan (Nishi, 1980).

Brassica rapa and $B$. juncea possess wide-ranging utility and highly diverse morphologies that make them important materials for plant genetic resources conservation and use. To conserve and use these plant genetic resources effectively, it is essential to develop markers that not only distinguish individuals and accessions but also reflect the inherent diversity and relationships among collection holdings (Kresovich and McFerson, 1992). Random amplified polymorphic DNA (RAPD) markers have been used to characterize identities and relationships within Brassica oleracea. Kresovich et al. (1992) showed that these markers can be of great value in genetic resources management as a quick, cost-effective, and reliable method for identification, measurement of variation, and determination of similarity at the intraspecific level. Although some workers (Demeke et al., 1992; Kresovich et al., 1992) have used RAPD markers to examine a few selected Chinese vegetable brassicas, until now, there has been no survey using RAPD markers that characterized a large number of $B$. rapa and $B$. juncea accessions.

This investigation used RAPDs to identify taxonomic groups and assess the relationships between and within selected accessions of Brassica rapa and B. juncea. The objectives were to 1) determine if it was possible to generate a sufficient number of RAPD markers to discriminate among the accessions, the subspecies, and the species of B. rapa, B. juncea, and B. oleracea that were included in this study; 2) determine if any of the RAPD markers were invariable at the accession, subspecies, or species level, and if so, to determine if subsets of invariable fragments were diagnostic, that is, if they provided unique DNA profiles at specific taxonomic ranks that would unambiguously identify taxa; 3) use RAPD markers to determine relationships within and between accessions, subspecies, and species, using similarities computed from RAPD marker profiles; 4) establish a baseline of relationships between accessions of the Chinese vegetable brassicas that could serve as a starting point for assessing the diversity within the national collection of this material and for judging the distinctiveness of accessions being considered as future additions to the collection; and 5) test whether B. rapa ssp. pekinensis was more likely to have originated by hybridization between $B$. rapa ssp. chinensis and $B$. rapa ssp. rapifera or by selection and/or differentiation of either B. rapa ssp. chinensis or B. rapa ssp. rapifera alone.

\section{Materials and Methods}

Plant materials. All 52 accessions analyzed originated in China (Table 1). The study included 30 accessions of $B$. rapa representing four ssp., 18 accessions of $B$. juncea representing three ssp. and four accessions of B. oleracea representing one ssp. These materials were selected for examination because they represent a diverse array of Chinese vegetable Brassica accessions that have economic importance, encompass a wide range of phenotypic diversity, have varied end use, and require complex ex situ maintenance because of their large plant size, vernalization requirement, allogamous breeding system, and limited viability in storage.

Ten seeds per accession were sown in Cornell Mix soil $1 \mathrm{~N}-1 \mathrm{P}$ $0.5 \mathrm{~K}$ in 5 -cm-diameter pots in the greenhouse on 9 Nov. 1992.
Plants were transplanted into 10 -cm-diameter pots on 1 Dec. 1992. Greenhouse temperature was regulated to $25 \mathrm{C}$ during the day and $18 \mathrm{C}$ during the night. Pesticide spraying was conducted once a week, following standard practice.

Genomic DNA extraction. After 60 days of plant growth, for each individual plant the four or five youngest healthy leaves were harvested, placed into coin envelopes, and immediately frozen by dipping into liquid $\mathrm{N}_{2}$. Tissue was then freeze-dried and stored at 20C until use. DNA was extracted from one to four plants (Table 1 ) per accession. Extractions from four individual plants per accession allowed for the assessment of within-accession variability.

Tissue grinding followed the protocol of Colosi and Schaal (1993), with 0.06 to $0.10 \mathrm{~g}$ of freeze-dried tissue and $1.5-\mathrm{ml}$ microfuge tubes. Immediately after grinding, genomic DNA extractions were performed according to the protocol of Hillis et al. (1990). The final DNA pellet was redissolved in $100 \mu \mathrm{l}$ TE solution (10 mM Tris- $\mathrm{HCl}, \mathrm{pH} 7.5$, and $0.1 \mathrm{~mm}$ EDTA), and the DNA concentration was determined according to the manufacturer's instructions using a DNA fluorometer (model TKO 100; Hoefer Scientific Instruments). All DNA samples were diluted to a standard concentration of $5 \mathrm{ng} \cdot \mathrm{ml}^{-1}$ before use.

$R A P D$ assay. Following an initial screening of 20 decamer oligonucleotide primers (synthesized by Operon Technologies, Alameda, Calif.), the five primers that showed the greatest number of polymorphisms were selected for use in amplification. The DNA amplification protocol reported by Williams et al. (1990) was used with two modifications described by Kresovich et al. (1992): 1) temperature was set at $38 \mathrm{C}$ during the annealing stage of the amplification cycle, 2) annealing time was increased to $5 \mathrm{~min}$.

Reaction mixtures $(25 \mu \mathrm{l})$ contained $10 \mathrm{~mm}$ Tris- $\mathrm{HCl}(\mathrm{pH} 8.3)$; $50 \mathrm{~mm} \mathrm{KCl} ; 2.0 \mathrm{~mm} \mathrm{MgCl}$; $0.001 \%$ gelatin; $0.2 \mathrm{~mm}$ each of dATP, dCTP, dGTP, and dTTP (Pharmacia LKB Biotechnology, Piscataway, N.J.); $0.64 \mu \mathrm{M}$ of a single decamer primer; about 25 ng of genomic DNA; and 0.625 unit of DNA Thermus aquaticus (Taq) polymerase (Perkin Elmer Cetus, Norwalk, Corm.). Reaction mixtures were incubated in a DNA 9600 thermocycler (Perkin Elmer Cetus) programmed for 45 cycles of $1 \mathrm{~min}$ at $94 \mathrm{C}$, $5 \mathrm{~min}$ at $38 \mathrm{C}$, a $3-\mathrm{min}$ ramp to $72 \mathrm{C}$, and $2 \mathrm{~min}$ at $72 \mathrm{C}$. The same reaction mixture without genomic DNA was run with each amplification to serve as a negative control. Two replicate amplification products per sample were produced and analyzed by gel electrophoresis in $2 \%$ agarose in $1 x$ TBE. Sizes of the amplification products were estimated using a l-kb DNA ladder (BRL, Bethesda, Md.). The gels were stained with ethidium bromide and photographed with black and white film \#667 (Polaroid, Cambridge, Mass.) over UV light. Each amplified fragment was identified by the primer used to produce it and its approximate size in base pairs.

Data analysis. Similarities were computed by the method of Nei and Li (1979) and were corrected for false positives and false negatives by the method of Lamboy (1994b), weighting false positives and false negatives equally. False positives are bands that are present, but which ancillary information, such as known pedigrees or replicate runs, suggests may be PCR artifacts; false negatives are bands that are not present, but which supplementary information indicates should be observed (Lamboy, 1994a). Although it is not possible in general to determine the specific bands that are false positives and false negatives (Lamboy, 1994a), it is possible to determine the probabilities with which these artifacts occur in a specific data set (Lamboy, 1994b). Similarities were calculated and fingerprinting was done using programs written by W.F. Lamboy in the GAUSS programming language (Edlefsen and 
Jones, 1986). Phenograms were determined by the UPGMA method (Sneath and Sokal, 1973) using the program NTSYS (Rohlf, 1988).

DNA profiling. DNA profiles (set of invariable fragments) for each accession, each subspecies, and each species were obtained and compared to one another to assess whether any sets of fragments were diagnostic, i.e., could unambiguously identify a taxon at a specific taxonomic rank.

Since each DNA sample was amplified twice, fragments were considered to be present only if both DNA amplifications produced a fragment. Fragments were considered to be absent only if both DNA amplifications lacked the fragment. Fragments were said to be invariable at the accession level if the fragments were either present in all plants belonging to the accession or absent in all plants belonging to the accession. Invariable fragments were defined analogously for subspecies and species. If a fragment was present in one amplification and absent in the other, the fragment was considered to be variable in that DNA sample. It should be noted that a particular RAPD fragment could be invariable in one accession, subspecies, or species, but variable in another. Fragments that were variable across amplifications of DNA from a single plant were used in computing estimates of false positives and false negatives as described in Lamboy (1994b).

The discovery of fragments that were diagnostic for a particular taxon at a particular taxonomic level was a two-step process. The first step was to find a set of invariable fragments for each taxon. The second step was to compare the sets of invariable fragments for all pairs of taxa (at a specific taxonomic rank) and to determine if any of the taxa could be unambiguously distinguished from the others. A set of invariable fragments that distinguished two taxa from each other was considered to be diagnostic for the comparison of the two taxa. A set of fragments rather than a single fragment was used to distinguish taxa because RAPD analyses of additional plants of a taxon may show that a RAPD band that appeared to be invariable when the original sample was analyzed may turn out to be variable when additional plants of that taxon are included. Distinguishing taxa by the maximum number of invariable fragments increases the chances that they will remain distinguishable even after additional DNA samples are analyzed.

Table 2. An example of fragments that distinguish between two accessions, No. 13 and No. 20, of Table 1; $0=$ fragment is never present in the species, $1=$ fragment is always present in the species. Fragments that do not distinguish the pair of accessions (and are not listed) are either always present in both accessions, always absent in both accessions, or sometimes present and sometimes absent in one or both accessions. Note that for any pair of accessions selected from the 52 analyzed there exist RAPD markers that distinguish between them.

\begin{tabular}{lcc}
\hline \hline Fragment no. & Accession 13 & Accession 20 \\
\hline X04-1630 & 0 & 1 \\
X04-1430 & 0 & 1 \\
X04-890 & 0 & 1 \\
X04-620 & 0 & 1 \\
X06-970 & 0 & 1 \\
X06-700 & 1 & 0 \\
X06-470 & 1 & 0 \\
X07-1960 & 1 & 0 \\
X07-1640 & 1 & 0 \\
X07-770 & 0 & 1 \\
X16-1230 & 0 & 1 \\
X17-560 & 0 & 1 \\
X17-420 & 1 & 0
\end{tabular}

\section{Results and Discussion}

Five of the 20 primers tested generated a sufficient number of RAPD markers (112) for the analysis of accessions, subspecies, and species. Over 20 markers were produced by each primer. Probably no more than $5 \%$ of these fragments represented the chloroplast and mitochondrial genomes (Thormann and Osbom, 1992).

From the 112 RAPD markers, sets of fragments were found that were invariable for each accession, and subsets of these invariable fragments were found that were diagnostic for each accession. Thus, all 52 accessions could be distinguished from every other accession by means of a combination of fragments that were always present in one accession and always absent in the other. An example is shown in Table 2.

Although sets of fragments that were diagnostic for each accession were found, no sets of fragments were able to distinguish between subspecies within a species. Given the long history of cultivation and the intentional or accidental crossing of these subspecies, this result is not surprising. Nevertheless, it suggests that none of the subspecies considered originated as selections from other subspecies. If they had, one would expect to find bands distinguishing the descendant from the ancestor, assuming, of course, they there had been enough time for the divergence of the subspecies involved.

The species themselves, however, could easily be distinguished from one another using the RAPD markers shown in Table 3. Some fragments were useful in distinguishing between all three species, while others were only useful if two particular species needed to be distinguished.

Relationships between species (Table 4, Fig. 1) were determined by UPGMA cluster analysis of Nei-Li similarities. Similarities were corrected for artifactual fragments by the method of Lamboy (1994b) based on the estimated probabilities of false positives $=0.0092$ and false negatives $=0.0055$. Similarity results at the species level must be interpreted with caution, since it has been shown (Thormann and Osborn, 1992) in a study of $B$. oleracea, B. rapa, B. napus, B. nigra, B. carinata, and B. juncea that up to $20 \%$ ( 3 of 15 ) of the RAPD fragments that migrated to the same position on the gel and were scored as being homologous did not represent the same DNA sequence in different species.

Table 3. RAPD markers that are diagnostic for species: fragments present in the three species, that, in combination, distinguish each species from the other two. $0=$ Fragment is never present in the species, $1=$ fragment is always present in the species, ${ }^{*}=$ fragment is sometimes present and sometimes absent in the species.

\begin{tabular}{lccc}
\hline \hline Fragment & Brassica rapa & Brassica juncea & Brassica oleracea \\
\hline \hline X04-950 & $*$ & 1 & 0 \\
X04-920 & 0 & 0 & 1 \\
X04-560 & 1 & $*$ & 0 \\
X06-770 & 1 & 1 & 0 \\
X07-1140 & $*$ & 1 & 0 \\
X07-770 & $*$ & 1 & 0 \\
X07-400 & 1 & 1 & 0 \\
X16-2050 & 1 & 0 & 0 \\
X17-1480 & $*$ & 0 & 1 \\
X17-1320 & $*$ & 0 & 1 \\
X17-760 & 1 & 1 & 0 \\
X17-560 & $*$ & 0 & 1 \\
X17-420 & 0 & 1 & 0 \\
X17-390 & 0 & $*$ & 1 \\
\hline
\end{tabular}


Table 4. Between-species Nei-Li similarities with within-species similarities on the diagonal.

\begin{tabular}{|c|c|c|c|c|}
\hline & & rassica rapa & Brassica juncea & Brassica oleracea \\
\hline Brassica & rapa & 0.764 & & \\
\hline Brassica & uncea & 0.515 & 0.798 & \\
\hline Brassica & oleracea & 0.386 & 0.358 & 0.766 \\
\hline
\end{tabular}

Such fragments could inflate the values of the computed similarity coefficients. Nevertheless, the similarity of B. rapa and B. juncea was $52 \%$ using RAPD data, which is consistent with the origin of $B$. juncea as an allopolyploid derivative of $B$. rapa and $B$. nigra. Based on this known relationship of $B$. rapa and $B$. juncea, one would predict the Nei-Li similarity between B. rapa $(2 n=20)$ and $B$. juncea $(2 n=36)$ to be $20 / 36=5 / 9=0.55$ (assuming each chromosome possessed the same average number of RAPD markers). The observed value of 0.52 is extremely close to the expected value. Sampling error, linkage of markers, or changes in one or both of the genomes since the allopolyploid arose might account for the observed similarity being somewhat less than expected. In the case of $B$. rapa and $B$. juncea, even if there were nonhomologous bands that were incorrectly called homologous, there were not enough of them to inflate the similarity above the expected value.

The UPGMA analysis of similarities between subspecies (Table 5, Fig. 2) showed that in this study RAPD markers correctly group together subspecies belonging to a single species. Subspecies within a single species were always $70 \%$ similar or more. Subspecies belonging to $B$. rapa had similarities with subspecies belonging to $B$. juncea in the range $0.496-0.542$. These values are close
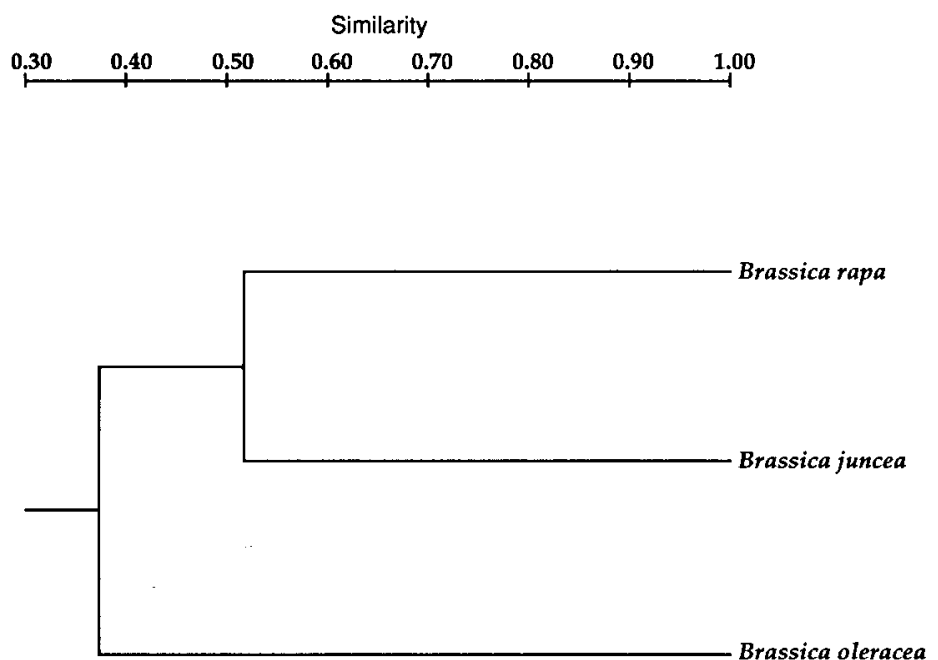

Fig. 1. Relationships between Brassica species as determined by UPGMA analysis using Nei-Li similarities computed from RAPD data. to those predicted based on the knowledge that $B$. juncea arose by hybridization of $B$. rapa and B. nigra.

The use of UPGMA to analyze the Nei-Li similarities produced clusters of accessions that belong to a species (Fig. 3). Individual accessions assigned to the same subspecies cluster fairly closely, although there are some exceptions. For example, one accession of $B$. juncea ssp. rapifera (59) clusters most closely with an accession of $B$. juncea ssp. multiceps (48). This is not unexpected, however, in a species where intentional and inadvertent crossing between subspecies has taken place.

Other pairs of accessions about which more information is known show interesting similarity relationships. For example, accessions 84 and 86 , have a similarity of 0.911 , but have dramatically different morphologies. Plants of accession 84 are fluffy topped with spreading leaves, while plants of accession 86 forma head with" strongly overlapping leaves. In a contrasting situation, seeds of accessions 24 and 84 were collected from two different villages in the Beijing area and the plants are called by the same name, yet the similarity between them was only 0.772 . Brassica rapa ssp. pekinensis accessions 20 and 23 are from different provinces and are morphologically very different, but have a similarity of 0.945 , the highest between any two $B$. rapa ssp. pekinensis accessions. The three pekinensis accessions, 29,30, and 31 , all come from two provinces near the coast (Hebei and Tianging) and have a cylindrical morphology, yet surprisingly they become part of the same cluster only at a similarity of 0.75 . One might expect that accessions having close geographic affinities and a common morphology would be more similar than this. Finally, pekinensis accessions 33 and 34 cluster very closely together (0.939) and have the same morphology, but are called by different names and were acquired from widely distant provinces (Shandong and Henan). It is likely that the progenitor of accession 34 was introduced long ago from Shandong and its name changed, but it did not change much genetically.

The lack of correspondence found in this study between similarity, geography, and morphology is analogous to the results of isozyme analyses of $B$. oleracea accessions reported by Lamboy et al. (1994). In that study there were no significant correlations between morphology, geography, and relationships. This lack of correspondence is almost certainly a result of the long history of intentional hybridizations within and even between species, combined with the fact that it appears that probably only a few genes are responsible for the large effects that determine cultivar groups.

Plants of B. rapa ssp. pekinensis from accessions 26 and 38 were crossed at the Institute of Vegetables and Flowers, Beijing, to produce accession 86. According to the RAPD data, accession 86 is more similar to accession $26(0.835)$ and accession $38(0.784)$ than 26 and 38 are to each other $(0.734)$. This is what one would expect of an accession that was the hybrid of 26 and 38.

Accessions 2 and 4, belonging to B. rapa ssp. chinensis, were

Table 5. Between-subspecies Nei-Li similarities with within-subspecies similarities on the diagonal.

\begin{tabular}{lllcccccc}
\hline \hline Species & Subspecies & & 1 & 2 & 3 & 4 & 5 & 6 \\
\hline Brassica rapa & chinensis & 1 & 0.805 & & & & \\
& utilis & 2 & 0.765 & 0.805 & & & & \\
& pekinensis & 3 & 0.751 & 0.742 & 0.801 & & & \\
\multirow{2}{*}{ Brassica juncea } & rapifera & 4 & 0.718 & 0.710 & 0.756 & 0.858 & & \\
& rapifera & 5 & 0.496 & 0.511 & 0.509 & 0.525 & 0.875 & \\
& tsa-tsai & 6 & 0.511 & 0.531 & 0.506 & 0.512 & 0.797 & 0.833 \\
\multirow{2}{*}{ Brassica oleracea } & multiceps & 7 & 0.509 & 0.542 & 0.525 & 0.533 & 0.763 & 0.770 \\
\hline
\end{tabular}



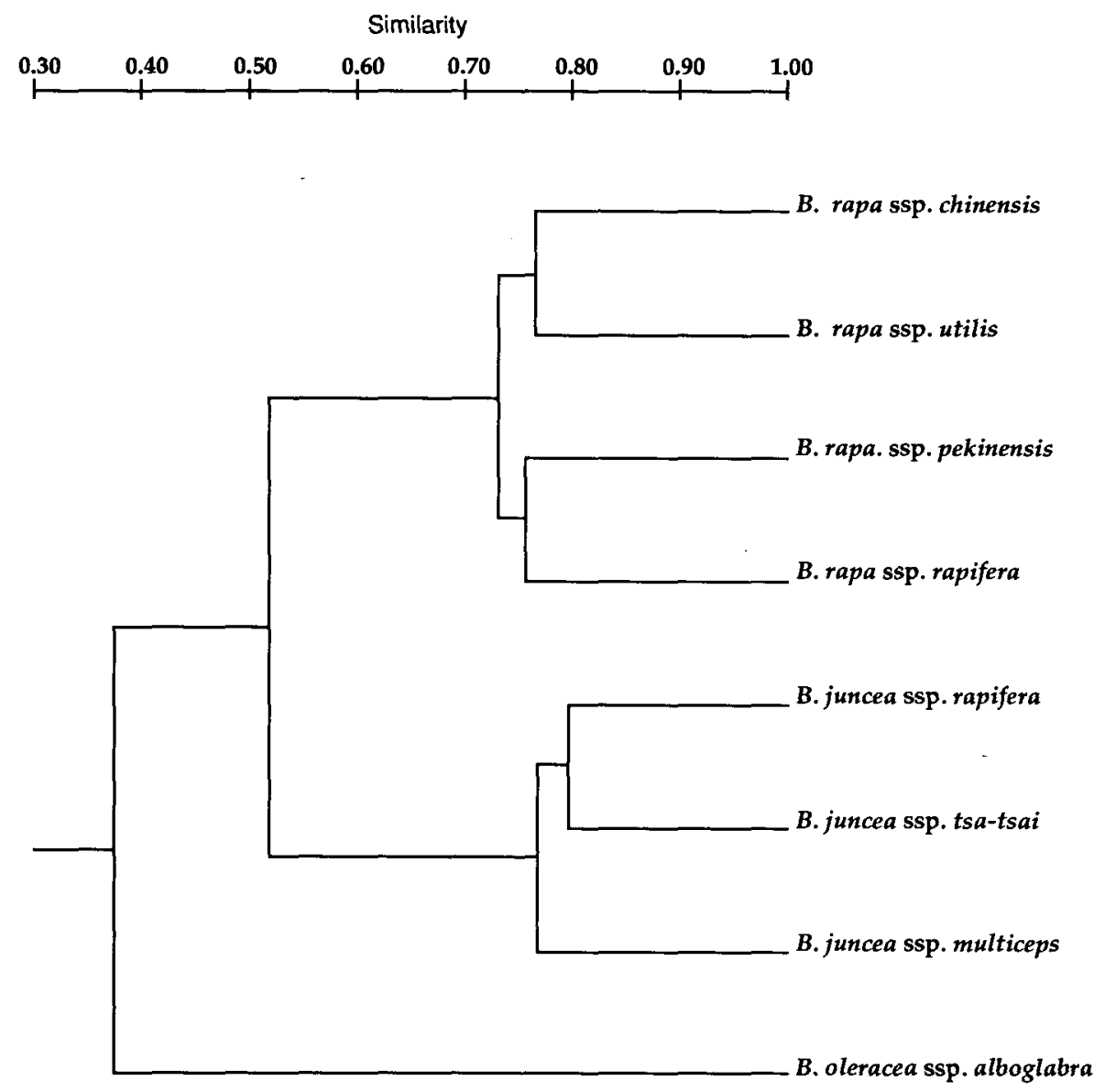

Fig. 2. Relationships between Brassica subspecies as determined by UPGMA analysis using Nei-Li similarities computed from RAPD data

both collected from Shanghai City are very similar morphologically, and their only major difference is the color of the petiole, whitish-green in 2 and dark green in 4 . Their similarity is 0.860 , although both are more closely related to other less morphologically similar accessions than to each other. Accessions 2 and 9, although from difference provinces, are not only closely related by similarity (0.861) but are morphologically different only in the petiole color ( 9 has a white petiole). The fact that these accessions of ssp. chinensis have different Chinese names (Table 1) suggests that the material was introduced to Beijing from Shanghai during historical times.

Accessions 42, 44, and 45 of Brassica juncea ssp. multiceps are leaf mustards having recompound leaves, and they are from three widely distant provinces. Yet all have similarities with one another in the range (0.834-0.860). Accessions 57 and 63, both root mustards having a similarity of 0.864 , are from different provinces, while accessions 69 and 75, both stem mustards having a similarity of 0.951 , are from the same province and are very similar morphologically. The similarities reflect the close geographic and morphological relationships of the two latter accessions.

RAPD data were collected for four different plants of one accession for each of the eight subspecies included in this study. The within-accession Nei-Li similarities show that the eight accessions are not uniformly diverse (Table 6). Four of the accessions showed no variability with respect to RAPD markers, while the other four accessions showed within-accession similarities ranging from 0.866 to 0.974 . The value of the within-accession similarity of accession 18 of $B$. rapa ssp. utilis of 0.866 is less than the within-subspecies similarity of subspecies $B$. juncea ssp. rapifera of 0.875 . Thus, in this particular data set, there is more variability $y$ within a single accession of a specific subspecies than in five accessions of another subspecies. This type of information can be critical for the effective and efficient maintenance of a genetic resources collection, since knowledge of the within-accession variability could strongly influence whether a particular accession were included in the collection. The value of a germplasm collection depends not only on the number of accessions it contains, but also upon the diversity present in those accessions. In this example, one would be able to preserve a greater level of diversity by the judicious selection of a single accession of one subspecies than by uncritical preservation of five accessions of another subspecies.

The RAPD marker data that have been collected forma baseline for the assessment of diversity in the current Chinese Brassica collection and for evaluation of potential new accessions. For example, when an accession is evaluated for possible incorporation into the collection, Nei-Li similarities between the new accession and existing accessions can be computed after information on the presence and absence of RAPD markers is obtained. These similarities can be used along with other types of information for including or excluding the possible addition to the collection. The baseline information on similarities provides a rational, objective means for evaluating potential additions to a genetic resources collection, particularly since the similarities are based on genetic information that is comparable across all existing and future accessions. This contrasts with the difficulties encountered when evaluating accessions for morphological characters, when quantitative measurements of characters are not readily obtained or are subject to varying environmental conditions.

Determination of RAPD similarities allowed for consideration of the three hypotheses of the origin of Brassica rapa ssp. pekinensis 


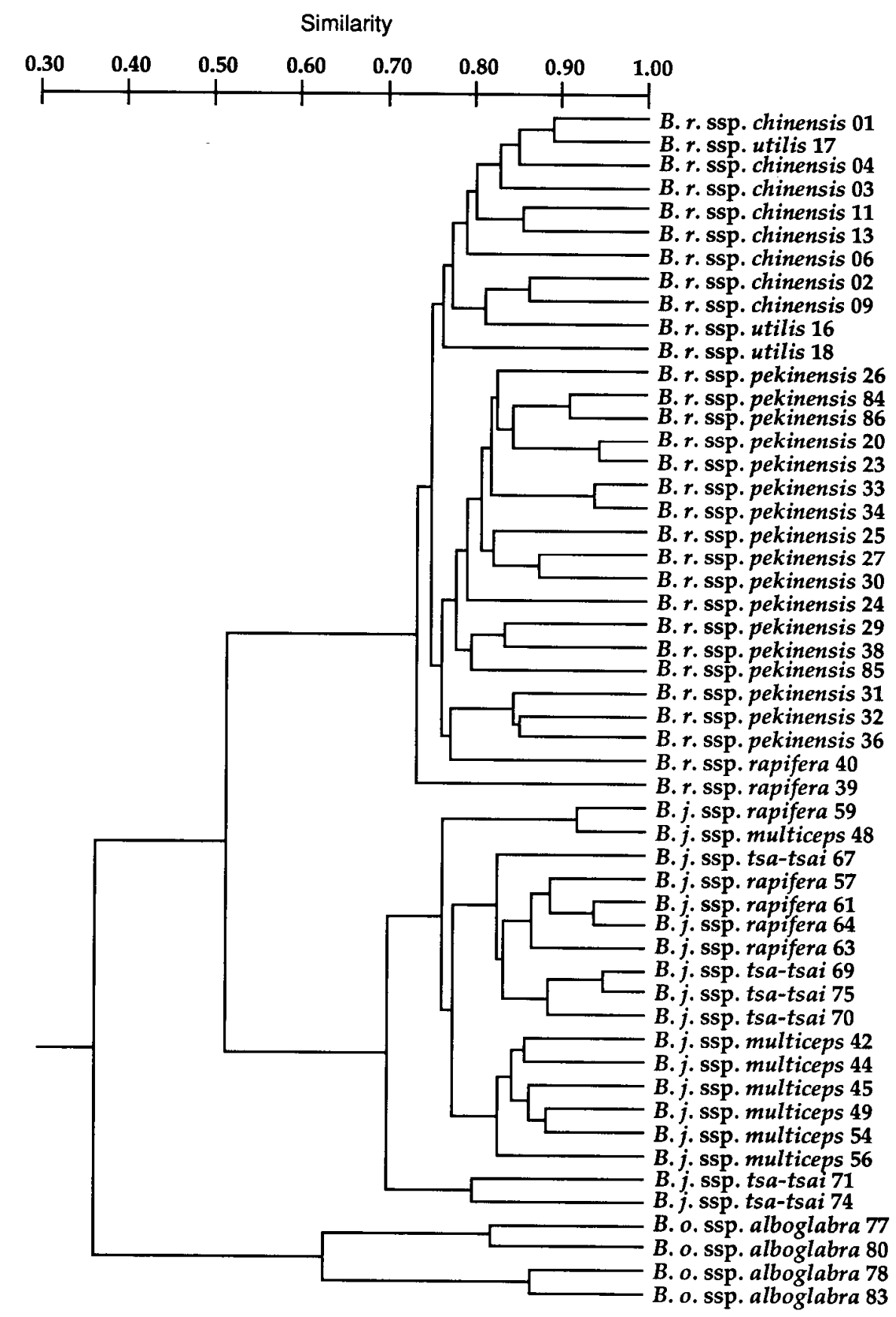

Fig. 3. Relationships between Brassica accessions as determined by UPGMA analysis using Nei-Li similarities computed from RAPD data

(Table 7). Expected similarities were computed between ssp. pekinensis, ssp. chinensis, and ssp. rapifera under the hypotheses that pekinensis arose from chinensis alone, rapifera alone, or as their hybrid. The differences between the actual and expected similarities of pekinensis and its putative progenitors, chinensis and rapifera, have the smallest magnitudes $(-0.011,-0.032)$ for the hypothesis that pekinensis arose as a hybrid. The differences are larger for the hypotheses that pekinensis arose by selection and differentiation of either ssp. chinensis $(-0.054,0.038)$ or ssp. rapifera $(0.033,-0.102)$. This suggests that pekinensis arose as a hybrid of ssp. chinensis and ssp. rapifera, rather than as a selection from chinensis or rapifera. The actual similarities between pekinensis and its two putative progenitors are less than expected, however, this does not invalidate the hypothesis of hybrid origin, since one would expect some evolutionary changes in the genomes of all three subspecies since the time that pekinensis arose. (Al- though the logical and statistical assumptions for a valid chi-square test of these differences are not satisfied, if one carries out the test anyway, one finds that none of the three hypotheses of origin can

Table 6. Within-accession similarities of accessions for which DNA was sampled from four plants.

\begin{tabular}{lcc}
\hline \hline Subspecies & Accession & Nei-Li similarity \\
\hline Brassica rapa ssp. chinensis & 04 & 1.000 \\
Brassica rapa ssp. utilis & 18 & 0.866 \\
Brassica rapa ssp. pekinensis & 20 & 1.000 \\
Brassica rapa ssp. rapifera & 39 & 0.914 \\
Brassica juncea ssp. multiceps & 49 & 1.000 \\
Brassica juncea ssp. rapifera & 57 & 0.974 \\
Brassica juncea ssp. tsa-tsai & 67 & 0.959 \\
Brassica oleracea ssp. alboglabra & 78 & 1.000 \\
\hline
\end{tabular}


Table 7. Test of three different hypotheses of the origin of Brassica rapa ssp. pekinensis. Hypotheses having smaller magnitude of differences between expected and actual are supported more strongly than hypotheses having greater magnitude of differences between expected and actual. This data most strongly supports the hypothesis that $B$. rapa ssp. pekinensis originated as a hybrid between ssp. chinensis and ssp. rapifera rather than the hypotheses that ssp. pekinensis arose as a selection from either ssp. chinensis or ssp. rapifera.

\begin{tabular}{|c|c|c|c|c|c|c|}
\hline \multirow{3}{*}{$\begin{array}{l}\text { If pekinensis } \\
\text { originated from }\end{array}$} & \multicolumn{4}{|c|}{ Similarities } & & \\
\hline & \multicolumn{2}{|c|}{ Expected } & \multicolumn{2}{|c|}{ Actual } & \multicolumn{2}{|c|}{ Difference } \\
\hline & chinensis & rapifera & chinensis & rapifera & chinensis & rapifera \\
\hline chinensis alone & 0.805 & 0.718 & 0.751 & 0.756 & -0.054 & 0.038 \\
\hline rapifera alone & 0.718 & 0.858 & $0.75 \mathrm{I}$ & 0.756 & 0.033 & -0.102 \\
\hline chinensis $\mathrm{x}$ rapifera & 0.762 & 0.788 & 0.751 & 0.756 & -0.011 & -0.032 \\
\hline
\end{tabular}

be rejected at the $90 \%$. significance level. ) Additional confirmatory work of the type conducted by Song et al. (1993) on the origin of the amphidiploids, B. juncea, B. carinata, and B. napus, is needed to resolve the origin of Chinese cabbage.

In this study five primers provided enough RAPD markers for the analysis of 52 accessions belonging to 8 subspecies and 3 species. The 112 different fragments could be used in combination to unambiguously identify all 52 different accessions. Although no combinations of fragments could distinguish between subspecies within species, species were easily identified using sets of RAPD markers. Nei-Li similarities were computed at all taxonomic ranks, and the results can be used as a baseline of relationships for future diversity assessment of Brassica rapa and B. juncea. Final] y Chinese cabbage ( $B$. rapa ssp. pekinensis) is more likely to have originated by hybridization between turnip (B. rapa ssp. rapifera) and pakchoi (B. rapa ssp. chinensis) rather than as a selection from either turnip or pakchoi alone. Overall, RAPD markers provide a fast, efficient technique for diversity assessment that complements methods currently in use in genetic resources collections.

\section{Literature Cited}

Colosi, J.C. and B.A. Schaal. 1993. Tissue grinding with ball bearing and vortex mixer for DNA extraction. Nucleic Acids Res. 21:105 1-1 052.

Demeke, T., R.P. Adams, and R. Chibbar. 1992. Potential taxonomic use of random amplified polymorphic DNA (RAPD): A case study in Brassica. Theoretical Applied Genet. 84: 990-994.

Edlefsen, L.E. and S. D. Jones. 1986. GAUSS programming language manual. Aptech Systems, Kent, Wash.

Hillis, D. M., A. Larson, S.K. Davis, and E.A. Zimmer. 1990. Nucleic acids III: Sequencing, p. 318-370. In: D.M. Hillis and C. Moritz (eds.). Molecular systematic. Sinauer, Sunderland, Mass.

Kresovich, S. and J.R. McFerson. 1992. Assessment and management of plant genetic diversity: Conservation of intra- and interspecific variation. Field Crops Res. 29:185-204.

Kresovich, S., J.G.K. Williams, J.R. McFerson, E.J. Routman, B.A. Schaal. 1992. Characterization of genetic identities and relationships of Brassica oleracea $\mathrm{L}$. via a random amplified polymorphic DNA assay. Theoretical Applied Genet 85: 190-196.

Lamboy, W.F. 1994a. Computing genetic similarity coefficients from RAPD data The effects of PCR artifacts. PCR Methods and Applications 4:31-37.

Lamboy, W.F. 1994b. Computing genetic similarity coefficients from RAPD data Correcting for the effects of PCR artifacts caused by variation in experimental conditions. PCR Methods and Applications 4:38-43.

Lamboy, W. F., J.R. McFerson, A.L. Westman, and S. Kresovich. 1994. Application of isozyme data to the management of the United States national Brassica oleracea L. genetic resources collection. GRACE 41:99-108.

Lee, S.H. 1982. Vegetable crops growing in China. Scientia Hort. 17:201209

Li, C.W. 1981. Origin, evolution, taxonomy and hybridization of Chinese cabbage, p. 3-11. In: N.S. Talekar and T.D. Griggs (eds.). Chinese Cabbage. AVRDC, Taiwan.

Nei, M. and W.-H. Li. 1979. Mathematical model for studying genetic variation in terms of restriction endonucleases. Proc. Natl. Acad. Sci. USA 76:5267-5273.

Nishi, S. 1980. Differentiation of Brassica crops in Asia and breeding of 'Hakuran' a newly synthesized leafy vegetable, p. 133-150. In: S. Tsunoda, K. Hinata, and C. Gomez-Campo (eds.). Brassica crops and wild allies. Japan Sci. Soc. Press, Tokyo.

Opena, R. T., G.G. Kuo, J.Y. Yoon. 1988. Breeding and seed production of Chinese cabbage in the tropics and subtropics. Tech. Bul. 17. AVRDC, Taiwan.

Prakash, S. and K. Hinata. 1980. Taxonomy, cytogenetics and origin of crop brassicas, a review. Opera Botanica 55:1-59.

Rohlf, F.J. 1988. NTSYS-pc. Applied Biostatistics, Setauket, NY.

Sneath, P.H.A. and R.R, Sokal. 1973. Numerical taxonomy. W.H. Freeman and Co., San Francisco.

Song, K. M., K. Tang, and T.C. Osborn. 1993. Development of synthetic Brassica amphidiploids by reciprocal hybridization and comparison to natural amphidiploids. Theoretical Applied Genet. 86:811-821.

Ten, Q. M. 1980. Origin, distribution and evolution of Chinese cabbage. Chinese Agri. Sci. 13:1-8.

Thormann, C.E. and T.C. Osborn. 1992. Use of RAPD and RFLP markers for germplasm evaluation. p. 9-11. In: Applications of RAPD Technology to Plant Breeding, Joint Plant Breeding Symposia Series, Crop Science Society of America, American Society for Horticultural Science, and American Genetic Association, Minneapolis, Minn.

Tsunoda, S. 1980. Eco-physiology of wild and cultivated forms in Brassica and allied genera, p. 109-120. In: S. Tsunoda, K. Hinata, and C. Gomez-Campo (eds.). Brassica crops and wild allies. Japan Sci. Sot, Press, Tokyo.

U, N. 1935. Genomic analysis in Brassica with reference to the experimental formation of $B$. napus and peculiar mode of fertilization. Japan J. Bet. 7:389-452.

Williams, J. G. K., A. R. Kubelik, K.J. Livak, J.A. Rafalski, and S.V. Tingey. 1990. DNA polymorphisms amplified by arbitrary primers are useful as genetic markers. Nucleic Acids Res. 18:6531-6535. 